NASA Technical Memorandum 103194

AIAA-90-2375

\title{
Numerical Investigation of the Thermal Stratification in Cryogenic Tanks Subjected to Wall Heat Flux
}

Chin-Shun Lin

Analex Corporation

NASA Lewis Research Center

Fairview Park, Ohio

and

Mohammad M. Hasan

National Aeronautics and Space Administration

Lewis Research Center

Cleveland, Ohio

Prepared for the

26th Joint Propulsion Conference

cosponsored by the AIAA, SAE, ASME, and ASEE

Orlando, Florida, July 16-18, 1990

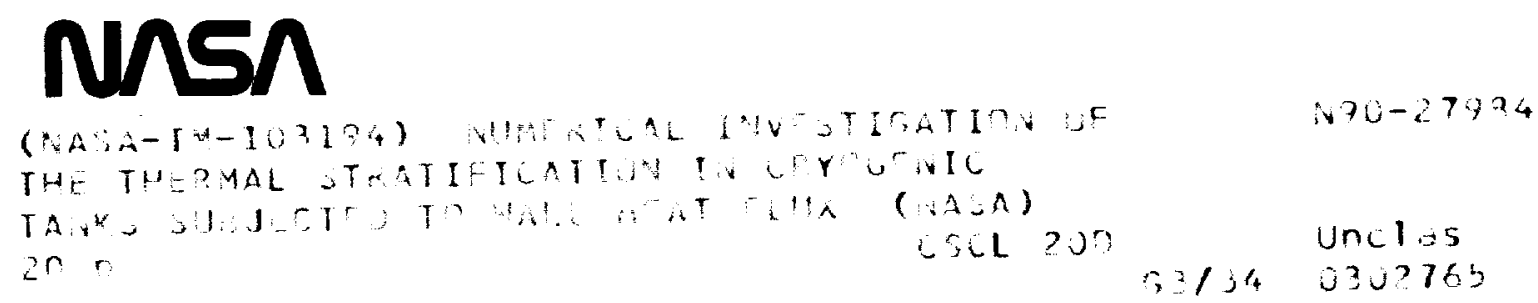





\title{
NUMERICAL INVESTIGATION OF THE THERMAL STRATIFICATION IN CRYOGENIC TANKS
}

\author{
SUBJECTED TO WALL HEAT FLUX \\ Chin-Shun Lin * \\ Analex Corporation \\ NASA Lewis Research Center \\ Fairview Park, Ohio 44126 \\ and \\ Mohammed M. Hasan ${ }^{\star \star}$ \\ National Aeronautics and Space Administration \\ Lewis Research Center \\ Cleveland, Ohio 44135
}

\section{ABSTRACT}

The flow pattern and thermal stratification of a cryogenic cylindrical tank are numerically studied. The tank sidewall is subjected to either a uniform heat-flux or two discrete levels of uniform heat-flux at the upper and lower halves of the tank wall. The tank bottom is kept at a constant temperature controlled by the heat exchanger of a thermodynamic vent system. The tank pressure is also assumed constant resulting in a constant saturation temperature at the interface which is higher than the tank bot tom temperature. The effects of vapor motion and vapor superheat on the mass and heat transfer processes at the interface are assumed negligible such that the calculations of liquid region can be decoupled from the vapor region. Dimensionless steadystate conservation equations are solved by a finite-difference method. The effects of modified Rayleigh number, Prandt 1 number, tank aspect ratio, wall heat-flux parameter, and wall heat-flux distribution on the liquid velocity and temperature fields are investigated. Also, their effects on the rate of heat transfer through the interface and the tank bottom are examined.

\section{NOMENCLATURE}

A surface area of the interface (cross-section area of the tank)

Ar aspect ratio, H/D

Bo tank Bond number, $\mathrm{ggD}^{2} / 4 \sigma$

$\mathrm{C}_{\mathrm{p}} \quad$ specific heat at constant pressure

D tank diameter

$\mathrm{Gr}^{*} \quad$ modified Grashof number, $\rho^{2} \beta g q_{w} \mathrm{H}^{4} / \mathrm{k \mu}^{2}$

\footnotetext{
${ }^{*}$ Research engineer, AIAA member.

**Aerospace engineer, AIAA member.
} 


\begin{tabular}{|c|c|}
\hline 8 & gravitational acceleration \\
\hline$g_{\mathbf{e}}$ & gravitational acceleration at the Earth surface \\
\hline $\mathrm{H}$ & liquid level height \\
\hline $\mathrm{h}_{\mathrm{fg}}$ & latent heat of evaporation \\
\hline $\mathrm{Ja}$ & Jakob number, $\mathrm{C}_{\mathrm{p}}\left(\mathrm{T}_{\mathrm{s}}-\mathrm{T}_{\mathrm{b}}\right) / \mathrm{h}_{\mathrm{fg}}$ \\
\hline k & thermal conductivity \\
\hline $\mathrm{m}_{\mathrm{e}}$ & evaporation mass flux \\
\hline $\mathrm{Nh}$ & wall heat-flux parameter, $q_{w} D / k\left(T_{S}-T_{b}\right)$ \\
\hline $\mathrm{Nh}_{\mathrm{l}}$ & defined as $q_{w, l} D / k\left(T_{s}-T_{b}\right)$ \\
\hline $\mathrm{Nh}_{\mathrm{u}}$ & defined as $q_{w, u} D / k\left(T_{s}-T_{b}\right)$ \\
\hline $\mathrm{Nu}_{t}, \mathrm{Nu}_{\mathrm{b}}$ & $\begin{array}{l}\text { local Nusselt numbers at the interface and tank bottom, } \\
\text { respectively }\end{array}$ \\
\hline $\mathbf{p}$ & pressure \\
\hline $\mathrm{pg}_{\mathrm{g}}$ & equilibrium hydrostatic pressure \\
\hline $\mathbf{p}^{*}$ & dimensionless pressure, $\left(p-p_{g}\right) / \rho(\alpha / D)^{2}$ \\
\hline $\operatorname{Pr}$ & Prandt l number, $\mathrm{C}_{\mathrm{p} \mu / \mathrm{k}}$ \\
\hline$q_{t}, q_{b}$ & $\begin{array}{l}\text { heat fluxes out of the liquid through the interface and tank } \\
\text { bottom, respectively }\end{array}$ \\
\hline $\mathrm{q}_{\mathbf{w}}$ & wall heat-flux for uniform heating or average wall heat-flux \\
\hline$q_{w, u}, q_{w, l}$ & $\begin{array}{l}\text { wall heat fluxes at the upper and lower halves of the tank wall, } \\
\text { respectively }\end{array}$ \\
\hline $\mathbf{r}$ & radial coordinate measured from the centerline \\
\hline$r^{\star}$ & dimensionless radial coordinate, $\mathrm{r} / \mathrm{D}$ \\
\hline $\mathrm{Ra}^{*}$ & modified Rayleigh number, $\mathrm{Gr}{ }^{\star} \mathrm{Pr}$ \\
\hline $\mathrm{R}_{\mathbf{q}}$ & $\begin{array}{l}\text { heat flux distribution parameter (the ratio of wall heat-flux } \\
\text { distribution), } q_{w, u} / q_{w, l}\end{array}$ \\
\hline $\mathrm{T}$ & temperature \\
\hline $\mathrm{T}_{\mathbf{s}}$ & interface temperature \\
\hline$T_{\mathbf{b}}$ & tank bot tom temperature \\
\hline
\end{tabular}




\begin{tabular}{ll}
$T^{*}$ & dimensionless temperature, $\left(T-T_{b}\right) /\left(T_{s}-T_{b}\right)$ \\
$u$ & axial velocity \\
$u^{*}$ & dimensionless axial velocity, $u /(\alpha / D)$ \\
$v_{S}$ & radial velocity \\
$v^{*}$ & radial velocity at the interface \\
$v_{S}^{*}$ & dimensionless radial velocity, $v /(\alpha / D)$ \\
$x$ & dimensionless radial velocity at interface \\
$x^{*}$ & axial coordinate measured from the tank bottom \\
$\alpha$ & dimensionless axial coordinate, $x / D$ \\
$\beta$ & thermal diffusivity, k/p $p$ \\
$\mu$ & coefficient of thermal expansion \\
$\rho$ & dynamic viscosity \\
$\sigma$ & liquid density \\
$\psi$ & surface tension \\
- & dimensionless stream function \\
\hline & average value over the interface or tank bot tom
\end{tabular}

\section{Subscripts}

b

l evaluated at the lower half of the tank wall

$s$ or $t$ evaluated at interface (top surface)

u

evaluated at the upper half of the tank wall

\section{INTRODUCTION}

Space missions in the coming decades will involve the storage and transfer of large volumes of cryogenic liquids such as hydrogen under orbital conditions. The current interest in cryogenic fluids storage and transfer stems in part from NASA's plan for an ambitious SEI (Space Exploration Initiative) including manned voyages to the moon and Mars. One of the key problems in storing cryogenic liquids under low-gravity conditions is the pressure control of storage tanks without significant loss of the fluid.

The thermal environment in space can result in heat transfer through an insulated cryogenic storage tank causing fluid temperature stratification and 
interfacial heat and mass transfer. As a consequence, the tank pressure is continually increasing. The prediction of the tank pressure $r$ ise rate and the technology development for the tank pressure and temperature control systems require knowledge of the temperature distribution in the liquid region. Generally, cryogenic storage systems of practical interest are dimensionally large. Therefore, the effect of natural convection on the thermal stratification process may be significant even in a micro-gravity environment. As for example, for a liquid hydrogen storage system, the modified Rayleigh number may vary in the range of $10^{5}$ to $10^{9}$ in an accelerational environment of $10^{-6} \mathrm{ge}$ or less. The problem described in this paper simulates some aspects of a cryogenic fluid storage system with a pressure control device such as a thermodynamic vent system (ref. 1).

The use of a thermodynamic vent system (TVS) is one of the major technologies (ref. 2) being developed by NASA for controlling cryogenic tank pressure. In a thermodynamic vent system, a small amount of cryogenic liquid is continuously withdrawn from the tank and passed through a Joule-Thomson valve resulting in a lower pressure and temperature two-phase fluid. The mixture is then introduced into a heat exchanger to either cool the tank liquid by absorbing its thermal energy and/or to intercept the heat leak which will otherwise be transferred into the liquid through the tank walls. The two-phase mixture is continually evaporating and the resulting vapor is then vented overboard. Therefore, if the TVS heat exchanger is mounted on some portion of the tank wall, the temperature of that portion of the tank wall will be relatively cold and constant because of the two-phase mixture of the TVS flow.

Generally, if the temperature of the lower portion of the tank is higher than that of the upper portion of the tank, buoyancy-induced mixing will be enhanced and the maximum temperature of the fluid is decreased. In this study, the tank pressure is assumed constant corresponding to a constant saturation temperature at the liquid-vapor interface. The TVS heat exchanger is assumed to be mounted on the tank bottom such that the tank bot tom temperature is lower than the interface temperature. This may represent the worst situation in which the maximum fluid temperature is increased. The tank sidewall is subjected to a prescribed heat flux. The heated liquid near the tank sidewall flows toward the interface under the action of the buoyancy force. A layer of highly stratified liquid is then formed underneath the interface at a temperature higher than the bulk liquid. At steady state, the energy coming through the tank sidewall must equal the total energy transferred out through the interface and the tank bottom. The objective of the present study is to numerically investigate the effects of buoyancy force, tank aspect ratio, wall heat-flux, and fluid thermodynamic properties on the rate of heat transfer through the free surface and the cold tank bottom. Their effects on the liquid velocity and the temperature fields are also examined.

\section{PROBLEM FORMULATION}

The physical system considered and coordinates are illustrated in figure 1. A circular cylindrical tank of diameter D is filled with a cryogen such as liquid hydrogen. nitrogen, or oxygen at a liquid level height $H$. The tank sidewall is subjected to either a uniform heat-flux $\left(q_{w}\right)$ or two discrete levels of uniform heat-flux $\left(q_{w, u}\right)$ and $\left(q_{w, l}\right)$ at the upper and lower 
halves, respectively, of the tank wall such that the average wall heat-flux,

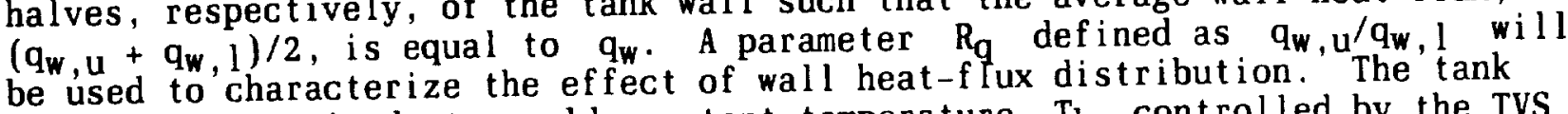
bot tom is maintained at a cold constant temperature $T_{b}$ controlled by the TVS heat exchanger. It is assumed that the effect of vapor superheat and vapor motion on the liquid-vapor interface heat and mass transfer is negligible. Thus, the calculations of the liquid region can be decoupled from the vapor region. The interface is assumed to be flat, wave free, and at a constant temperature $T_{S}$ corresponding to the constant tank pressure. It is further assumed that the change of liquid level due to the liquid consumption of the TVS and mass transfer at the interface is negligible.

Under the conditions and assumptions described above, the problem essentially reduces to a steady-state natural convection flow in an enclosure with zero friction at the free surface. The dimensionless form of constant-property continuity, momentum, and energy equations governing this axisymmetric laminar natural convection flow with Boussinesq approximation can be written as

$$
\begin{gathered}
\frac{\partial u^{\star}}{\partial x^{\star}}+\frac{\partial r^{\star} v^{\star}}{r^{\star} \partial r^{\star}}=0 \\
\frac{\partial u^{\star} 2}{\partial x^{\star}}+\frac{\partial u^{\star} r^{\star} v^{\star}}{r^{\star} \partial r^{\star}}=-\frac{\partial p^{\star}}{\partial x^{\star}}+\frac{R a^{\star} P r}{\operatorname{Nh} A r^{4}} T^{\star}+\operatorname{Pr}\left[\frac{\partial^{2} u^{\star}}{\partial x^{\star}}+\frac{\partial}{r^{\star} \partial r^{\star}}\left(r^{\star} \frac{\partial u^{\star}}{\partial r^{\star}}\right)\right] \\
\frac{\partial u^{\star} v^{\star}}{\partial x^{\star}}+\frac{\partial r^{\star} v^{\star} 2}{r^{\star} \partial r^{\star}}=-\frac{\partial P^{\star}}{\partial r^{\star}}-\operatorname{Pr} \frac{v^{\star}}{r^{\star} 2}+\operatorname{Pr}\left[\frac{\partial^{2} v^{\star}}{\partial x^{\star}}+\frac{\partial}{r^{\star} \partial r^{\star}}\left(r^{\star} \frac{\partial v^{\star}}{\partial r^{\star}}\right)\right] \\
\frac{\partial u^{\star} T^{\star}}{\partial x^{\star}}+\frac{\partial r^{\star} v^{\star} T^{\star}}{r^{\star} \partial r^{\star}}=\left[\frac{\partial^{2} T^{\star}}{\partial x^{\star 2}}+\frac{\partial}{r^{\star} \partial r^{\star}}\left(r^{\star} \frac{\partial T^{\star}}{\partial r^{\star}}\right)\right]
\end{gathered}
$$

where the nondimensionalized variables are defined as

$$
\begin{gathered}
\mathrm{x}^{\star}=\frac{\mathrm{x}}{\mathrm{D}}, \quad \mathrm{r}^{*}=\frac{\mathrm{r}}{\mathrm{D}}, \quad \mathrm{u}^{*}=\frac{\mathrm{u}}{(\alpha / \mathrm{D})}, \quad \mathrm{v}^{*}=\frac{\mathrm{v}}{(\alpha / \mathrm{D})} \\
\mathrm{T}^{\star}=\frac{\mathrm{T}-\mathrm{T}_{\mathrm{b}}}{\mathrm{T}_{\mathrm{s}}-\mathrm{T}_{\mathrm{b}}}, \quad \mathrm{p}^{\star}=\frac{\mathrm{p}-\mathrm{p}_{\mathrm{g}}}{\rho(\alpha / \mathrm{D})^{2}}
\end{gathered}
$$

Note that the gravity term has been substracted from the momentum equations by using the hydrostatic equilibrium equations. The parameters appearing in the governing equations are the Prandt $l$ number (Pr), the modified Rayleigh number $\left(\mathrm{Ra}^{*}\right)$, the wall heat-flux parameter $(\mathrm{Nh})$, and the tank aspect ratio (Ar):

$$
\begin{gathered}
\operatorname{Pr}=\frac{\mu}{\rho \alpha}=\frac{C_{p^{\mu}}}{k}, \quad R a^{*}=G r^{\star} \operatorname{Pr}=\frac{\rho^{2} \beta g q_{w} H^{4} C_{p}}{k^{2} \mu} \\
N h=\frac{q_{w} D}{k\left(T_{s}-T_{b}\right)}, \quad A r=\frac{H}{D}
\end{gathered}
$$


Boundary conditions are required to solve the governing partial differential equations (1) to (4). At the centerline, the symmetric conditions are applied:

$$
\frac{\partial u^{*}}{\partial r^{*}}=\frac{\partial T^{*}}{\partial r^{*}}=0, \quad v^{*}=0
$$

Nonslip conditions are used for the tank sidewall and bottom:

$$
\mathrm{u}^{\star}=\mathrm{v}^{\star}=0
$$

The thermal condition at the tank sidewall is either, for a uniform wall heat flux

$$
\frac{\partial T^{*}}{\partial r^{*}}=N h \text { at the entire tank sidewall }
$$

or, for two discrete levels of uniform wall heat fluxes,

$$
\frac{\partial T^{*}}{\partial r^{*}}= \begin{cases}N h_{u} & \text { at the upper half of the tank sidewall } \\ N h_{l} \text { at the lower half of the tank sidewall }\end{cases}
$$

where

$$
\mathrm{Nh}_{\mathrm{u}}+\mathrm{Nh}_{1}=2 \mathrm{Nh}
$$

The liquid-vapor interface and tank bottom are maintained at constant temperatures $T_{s}$ and $T_{b}$, respectively, with $T_{s}>T_{b}$. Thus,

$$
T^{*}=1 \quad \text { at } \quad x^{*}=\frac{H}{D}
$$

and

$$
\mathrm{T}^{*}=0 \text { at } \mathrm{x}^{*}=0
$$

The evaporation-induced (or may be condensation-induced) axial velocity at the interface is calculated by

$$
u_{e}=\frac{m_{e}}{\rho}=-\frac{k\left(\frac{\partial T}{\partial x}\right)_{s}}{\rho h_{f g}}
$$

where $m_{e}$ is the interface evaporation mass flux and $h_{f g}$ is the latent heat of evaporation. The nondimensional evaporation-induced velocity $u_{e}^{*}$ can be
expressed as

$$
u_{e}^{*}=-\mathrm{Ja}\left(\frac{\partial T^{\star}}{\partial x^{*}}\right)_{s}
$$


where $J a$ is the Jakob number defined by $C_{p}\left(T_{s}-T_{b}\right) / h_{f g}$. In practical applications, the value of Ja for liquid hydrogen, nitrogen, or oxygen, is small $(<0.1)$ and $u_{e}^{*}$ is generally negligible. Thus, it is assumed $u_{e}^{*}=0$ (i.e., Ja $=0$ ) in the present calculations. The shear-free condition is also applied at the interface since the vapor viscosity is usually much smaller than the liquid viscosity. Therefore, the following boundary conditions,

$$
u^{\star}=0, \quad \frac{\partial v^{\star}}{\partial x^{\star}}=0 \quad \text { at } \quad x^{\star}=\frac{H}{D}
$$

are used at the interface.

The energy balance in the liquid region can be expressed as

$$
\int q_{t} d A_{s}+\int q_{b} d A_{b}-q_{w} \pi D H=0
$$

where $q_{w}$ and $q_{b}$ are the incoming heat flux through the tank sidewall and the heat flux transferred out through the tank bottom, respectively, and $q_{t}$ is the heat flux transferred through the interface. The values of $q_{w}$ and $q_{b}$ are always taken positive. However, the value of $q_{t}$ is taken positive and negative for heat transferred out of the liquid and into the liquid, respectively. Equation (17) simply shows that the net heat transfer in the liquid region is zero. The average Nusselt numbers, $\overline{\mathrm{Nu}}_{t}$ and $\overline{\mathrm{Nu}}_{\mathrm{b}}$, at the tank interface and tank bottom, respectively, are defined as

$$
\overline{\mathrm{Nu}}_{t}= \pm \frac{\left(\frac{\overline{\mathrm{q}}_{t}}{\mathrm{~T}_{s}-\mathrm{T}_{b}}\right) \mathrm{D}}{k}
$$

and

$$
\overline{\mathrm{Nu}}_{\mathrm{b}}=\frac{\left(\frac{\overline{\mathrm{q}}_{\mathrm{b}}}{\overline{\mathrm{T}}_{\mathrm{s}}-\mathrm{T}_{\mathrm{b}}}\right) \mathrm{D}}{\mathrm{k}}
$$

where $\vec{q}_{t}$ and $\bar{q}_{b}$ are the average values of $q_{t}$, and $q_{b}$ over the surfaces of the interface and tank bottom, respectively. In equation (18), the positive sign is used if $\bar{q}_{t}>0$ and the negative sign is used if $\bar{q}_{t}<0$. By using the definitions of $\mathrm{Nh}$ and equations (18) and (19), equation (17) then becomes

$$
\overline{N u}_{b}=\left\{\begin{array}{lll}
4 N h A r-\overline{N u}_{t} & \text { if } & \bar{q}_{t}>0 \\
4 N h A r+\overline{N u}_{t} & \text { if } & \bar{q}_{t}<0
\end{array}\right.
$$

The ratio, $\overline{\mathrm{Nu}}_{\mathrm{b}} /(4 \mathrm{Nh} \mathrm{Ar})$, represents the fraction of the incoming energy transferred through the cold surface (i.e., removed by the TVS heat exchanger). If the value of $\overline{\mathrm{Nu}}_{\mathrm{b}} /(4 \mathrm{Nh} \mathrm{Ar})$ is greater, then the energy transferred to the 
interface to cause liquid vaporization is less. The energy ratio can be expressed as a function of the relevant parameters

$$
\frac{\overline{N u}_{b}}{4 N h A r}=f\left(R^{*}, \operatorname{Pr}, N h, A r, R_{q}\right)
$$

The effects of the above parameters on the ratio, $\overline{\mathrm{Nu}}_{\mathrm{b}} / 4 \mathrm{Nh} \mathrm{Ar}$, and the velocity and temperature fields are numerically investigated.

\section{COMPUTATIONAL METHOD}

The governing equations (1) to (4) are solved by a finite-difference method. A staggered grid system is employed with the scalar properties, $p^{*}$ and $T^{*}$, located midway between the $u^{*}$ and $v^{*}$ velocity grid nodes. The finite-difference equations are then derived by integrating the differential equations over an elementary control volume surrounding a grid node appropriate for each dependent variable. Bounded skew hybrid differencing (BSHD) (ref. 3) is used in the convective terms and the integrated source terms are linearized. Both of these practices enhance numerical stability. To accelerate convergence, pressures are obtained by using the pressure implicit split operator (PISO) method (ref. 4) to solve a pressure-correction equation which yields the pressure change needed to acquire velocity changes to satisfy mass continuity. The $f$ inite-difference equations are iteratively solved by the alternating direction implicit (ADI) method (ref. 5) with under-relaxation unt il the solutions are converged.

A nonuniform grid distribution is generated by using Roberts' transformation (ref. 6) with concentration of the grid nodes in the near-wall, nearbottom, and near-interface regions where the gradients of flow properties are large. Several grid distributions ( 36 by 20,48 by 27,60 by 34 , and 72 by 41) have been tried to obtain reasonable grid-independent solutions. It is found that the changes in the average radial velocity at the interface $\left(\bar{v}_{s}^{*}\right)$ and the average Nusselt number at the tank bottom ( $\left.\mathrm{Nu}_{b}\right)$ between grid distributions 60 by 34 and 72 by 41 are $<0.1$ percent for the worst case of uniformly-heated tank $\left(\mathrm{Nh}=4, \mathrm{Ar}=1.25\right.$, and $\left.\mathrm{Ra}^{\star}=10^{9}\right)$. Thus, it is decided to use 60 by 34 grid distribution for all of the calculations in the present study.

\section{RESULTS AND DISCUSSION}

Numerical solutions are obtained for the aspect ratio (Ar), the modified Rayleigh number $\left(\mathrm{Ra}^{*}\right)$, and the wall heat-flux parameter (Nh) ranging from 0.25 to $1.25,10^{5}$ to $10^{9}$, and 1 to 4 , respectively. The heat-flux distribution parameter $\left(R_{q}\right)$ is varied from 0 to 3 . The Prandt number (Pr) is varied from 1.25 to 2.6 to cover the typical values of $\mathrm{Pr}$ for liquid hydrogen, nitrogen,

\section{Effects of $\mathrm{Ra}^{*}$ and $\mathrm{Pr}$}

The effects of the modified Rayleigh number ( $\left.\mathrm{Ra}^{\star}\right)$ on the flow pattern are shown in figures 2 (a) to (C) for a uniformly-heated tank with $\operatorname{Ar}=1$, Nh $=4$, 
and $\operatorname{Pr}=1.25$. The dimensionless stream function, $\psi$, in the figure is calculated from

$$
\begin{gathered}
\psi\left(x^{*}, r^{*}=0\right)=\psi\left(x^{*}=0, r^{*}=0\right)-\int_{0}^{x^{*}} r^{*} v^{\star} d x^{*} \\
\psi\left(x^{\star}, r^{\star}\right)=\psi\left(x^{*}, r^{*}=0\right)+\int_{0}^{r^{*}} r^{*} u^{\star} d r^{*}
\end{gathered}
$$

with $\psi\left(x^{\star}=0, r^{*}=0\right)$ set to zero. For $\mathrm{Ra}^{\star}=10^{5}$ (fig. $\left.2(\mathrm{a})\right)$, the small velocity field forms a big and relatively symmetric counter-clockwise vortex flow pattern and the heat transfer is dominated by both convection and conduction. As $\mathrm{Ra}^{*}$ increases, a secondary flow of small clockwise vortex is generated in the lower left corner (fig. 2(b)) forcing the center of the counter-clockwise vortex moving toward the upper right corner and resulting in a narrower area for the boundary layer flow to turn radially toward the central region. The fluid velocity in the boundary and near the interface region is greater for larger $\mathrm{Ra}^{\star}$. Even though the radial flow turning area near the interface is smaller for higher $\mathrm{Ra}^{*}$, the returning flow velocity at the centerline is still larger than that for lower $\mathrm{Ra}^{\star}$. The clockwise vortex is lifted detaching from the tank bottom as $\mathrm{Ra}^{*}$ further increases and a thin boundary layer along the tank sidewall is developed (fig. 2(c)).

Without buoyancy force $\left(\mathrm{Ra}^{*}=0\right)$, there will be no fluid motion and the temperature distribution will result from the heat conduction only. As $\mathrm{Ra}^{*}$ increases, the natural covection process due to buoyancy results in warm fluid moving upward along the tank sidewall which increases the rate of heat transfer out of the interface. However, an increase in $\mathrm{Ra}^{*}$ convects the heat from the near-wall region faster. This results in lower and more uniform liquid temperature in the near-interface region (as seen from figs. 2 and 3 ) which decreases heat transfer rate at the interface. These two compensating effects result in the energy ratio, $\overline{\mathrm{Nu}}_{\mathrm{b}} /(4 \mathrm{Ar} \mathrm{Nh})$, only slightly changing with $\mathrm{Ra}^{*}$ for $\mathrm{Ra}^{\star}=10^{5}$ to $10^{9}$ (as seen in $\mathrm{fig} .4$ ).

Figures $3(\mathrm{a})$ to (c) show isotherm plots $\left(\mathrm{T}^{\star}\right)$ for the cases corresponding to figure 2. Since buoyancy-induced flow for higher $\mathrm{Ra}^{\star}$ convects heat from the tank wall faster than lower $\mathrm{Ra}^{*}$, the maximum temperature is lower for higher $\mathrm{Ra}^{*}$ and is located at the tank sidewall closer to the free surface. A natural convection boundary layer is clearly formed for higher Ra ${ }^{*}$ with slight temperature variation outside of the boundary layer. Most of the region has temperature higher than the interface. The higher Ra* yields relatively more uniform liquid temperature and lower degree of superheat in the liquid region. Figure 4 shows the effect of $\mathrm{Ra}^{*}$ on $\mathrm{Nu}_{\mathrm{b}} /(4 \mathrm{Ar} \mathrm{Nh})$, which represents the fraction of the total incoming energy transferred through the cold surface (tank bottom), for a uniform wall heat-flux of $N h=4$, Pr $=1.25$, and $\mathrm{Ar}=1$. The energy ratio reduces from 0.44 for $\mathrm{Ra}^{*}=10^{\overline{5}}$ to 0.42 for $\mathrm{Ra}^{*}=10^{7}$ and then increases to 0.43 for $\mathrm{Ra}^{*}=10^{9}$. This variation in the energy ratio is nearly negligible. Calculation (not presented here) also shows $\overline{N u}_{b} /(4 \mathrm{Ar} N \mathrm{Nh})$ is equal to 0.56 for $\mathrm{Ra}^{*}=0$ (heat conduction only). Thus, the energy ratio $\mathrm{Nub}_{\mathrm{b}} /(4 \mathrm{Ar} \mathrm{Nh})$ is significantly reduced by the buoyancy effect at lower modified Rayleigh numbers. The above analys is is obtained under the assumption of a flat interface. If the change of $R^{*}$ is due to the change in gravity, the above qualitative conclusion may apply only when 
the configurations of the interface are approximately the same, i.e., the tank Bond number (Bo) is much greater than one.

In the present calculations, the Prandtl number is varied from 1.25 to 2.60. The solutions (not presented in the paper) show that $\operatorname{Pr}$ has only a slight effect on the flow pattern and temperature distributions. A higher value of $\mathrm{Pr}$ enhances the buoyancy effect, resulting in greater radial velocity at the interface. Thus, the maximum temperature, located at the tank sidewall closer to the interface, decreases with increasing Prandt l number. Following the above observation, the energy ratio, also seen from figure 4 , decreases from 0.43 for $\operatorname{Pr}=1.25$ to 0.41 for $\operatorname{Pr}=2.65$ in a uniformly-heated tank with $\mathrm{Ar}=1.0$ and $\mathrm{Ra}^{*}=10^{6}$. Therefore, even though an increase in $\operatorname{Pr}$ transfers more of the incoming energy into the near-interface region, the degree of superheat in the liquid is reduced.

\section{Effects of $\mathrm{Nh}$ and $\mathrm{Ar}$}

An increase in the wall heat-flux parameter, Nh, can be obtained either by increasing the wall heat flux, $q_{w}$, or by decreasing the temperature difference, $\left(T_{s}-T_{b}\right)$. Higher $q_{w}$ yields warmer fluid in the natural convection thermal boundary layer moving toward the interface. A lower value of temperature difference, $\left(T_{s}-T_{b}\right)$, reduces the heat transferred to the cold bot tom. Both of the above effects tend to have more of the incoming energy transferred to the near-interface region. Therefore, the energy ratio, $\overline{\mathrm{Nu}}_{\mathrm{b}} /(4 \mathrm{Ar} \mathrm{Nh})$, decreases significantly with increasing Nh. Figure 5 shows the variation of the energy ratio as a function of the wall heat-flux parameter, $\mathrm{Nh}$, for $\mathrm{Ra}^{*}=10^{6}$. Also, for comparison, the pure conduction solution $\left(i . e ., \mathrm{Ra}^{*}=0\right)$ is included in the figure. For a given $\mathrm{Ra}^{\star}$, if $\mathrm{Nh}$ is small enough, the buoyancy effect on the energy ratio is negligible. For $\mathrm{Pr}=1.25, \mathrm{Ar}=1$, and $\mathrm{Ra}^{\star}=10^{6}$, the buoyancy has no effect on the energy ratio for $\mathrm{Nh}=1$ as seen from figure 5 . This critical Nh decreases with increasing $\mathrm{Ra}^{\star}$.

A lower value of $\mathrm{Nh}$ also yields smaller temperature difference between the tank wall and the bulk fluid, i.e., the maximum temperature is decreasing with decreasing Nh. This results in low velocity buoyancy-induced boundary layer flow and a weaker counter-clockwise vortex. Since the momentum of the returning flow at the centerline region is not strong enough to overcome the axial adverse pressure gradient, a small separation bubble is formed in the middle of the centerline. The returning flow moves radially toward the tank wall and feeds the boundary layer, resulting in the counter-clockwise vortex confined only in the upper portion of the tank. Figures 6(a) and (b) show that the lower half of the tank is nearly a dead zone and is dominated by heat conduction for a value of $\mathrm{Nh}=1$.

The effect of the tank aspect ratio (Ar) on the flow pattern of a uniformly-heated tank is shown in figures 7 (a) to (d) for $\operatorname{Pr}=1.25, \mathrm{Nh}=4$ and $\mathrm{Ra}^{*}=10^{6}$. The flow pattern for $\mathrm{Ar}=1$ is shown in figure $2(\mathrm{~b})$. The counter-clockwise vortex becomes more symmetric, with the vortex center moving from the upper right corner toward the midheight of the liquid region, as Ar is increasing. Also, an opposite weak vortex is formed at the centerline region and moves quickly toward the tank bottom as Ar increases. 
Figures $8(\mathrm{a})$ to (d) show the effect of $\mathrm{Ar}$ on the temperature field. The maximum temperature located at the tank sidewall increases with Ar. Generally, the liquid near the interface region is superheated over the entire tank cross-section. However, if $A r$ is too small, the convection boundary layer will not be well developed because the distance between the tank bot tom and the interface is too short. Thus, only a small region confined in the upper right corner can be superheated. This phenomena is shown in figure 8(a) for $\mathrm{Ar}=0.25$. Figure 9 shows that a lower $\mathrm{Ar}$ results in greater value of $\overline{\mathrm{Nu}}_{\mathrm{b}}$ ' (4 $\mathrm{Ar} \mathrm{Nh}$ ). It is noted that if $\mathrm{Ar}$ is too small (e.g., $\mathrm{Ar}=0.25$ in $\mathrm{fig} .9$ ), heat is coming in through the interface instead of going out. Thus, the value of the energy ratio, $\mathrm{Nu}_{\mathrm{b}} /(4 \mathrm{Ar} \mathrm{Nh})$, can be greater than one.

\section{Effect of $R_{q}$}

In this study, calculations are also made to investigate the effect of the heat-flux distribution parameter, $R_{q}=q_{w}, q_{w, l}$, on the flow pattern and temperature fields. Figure 10 shows a significant decrease in the energy ratio, $\mathrm{Nu}_{\mathrm{b}} /(4 \mathrm{Ar} \mathrm{Nh})$, as $\mathrm{R}_{\mathrm{q}}$ increases. It is because more heating on the upper portion of the tank wall $\left(R_{q}>1\right)$ enhances the buoyancy effect and contributes more heat in the near interface region. If the lower portion of the tank wall has more heat flux coming in $\left(R_{q}<1\right)$, the fluid near the lower portion of the tank wall moves upward in the boundary layer and encounters a relatively low temperature region near the upper tank wall where the wall heat-flux is lower. This results in a mixing between the bulk liquid and the natural convection boundary layer. Thus, the heat transferred to the interface is reduced and $\overline{\mathrm{Nu}}_{\mathrm{b}} /(4 \mathrm{Ar} \mathrm{Nh})$ is increased. From this observation, one can expect that if the given average heat-flux can be arranged such that $R_{q}<1$, the tank pressure rise rate will be lower. This finding is also in agreement with the experimental work by Evans et al. (ref.7).

Figures $11(\mathrm{a})$ and (b) show the streamline plots for $\mathrm{R}_{\mathrm{q}}=1 / 3$ and 3 , respectively. The streamline plot for $R_{q}=1$ is shown in figure $7(\mathrm{c})$. As $R_{q}$ increases from $1 / 3$ to 3 , the center of counter-clockwise vortex moves toward the interface resulting in a higher radial interface velocity. The weak secondary vortex tends to detatch from the tank bottom. The isotherm plots for $\mathrm{R}_{\mathrm{q}}=1 / 3$ and 3 are shown in figures $12(\mathrm{a})$ and $(\mathrm{b})$, respectively. The plot for $R_{\mathrm{q}}=1$ is shown in figure $8(\mathrm{c})$. For $R_{\mathrm{q}}=1 / 3$, there are two peak temperatures at the tank wall with the lower peak being the maximum temperature. As $R_{q}$ increases, the value of lower peak decreases and the value of the higher peak increases becoming the maximum temperature. If $\mathrm{R}_{\mathrm{q}}$ is large enough, the lower peak disappears and the maximum temperature moves slightly upward. On the other hand, if $\mathrm{R}_{\mathrm{q}}$ is decreasing from $1 / 3$, the value of upper peak is decreasing and the lower peak keeps increasing. The upper peak then disappears when $\mathrm{R}_{\mathrm{q}}$ is small enough.

At the interface, the numerical solutions show the local value of $u_{e}^{*}$ is generally two orders of magnitude smaller than $v_{s}^{*}$ (except in the small regions near the tank sidewall and centerline), especially for large $\mathrm{Ar}$ and $\mathrm{Nh}$ and low $\mathrm{Ra}^{\star}$. Thus, it is justified that the neglect of $\mathrm{u}_{e}$ in the calculations is acceptable. For the problems considered in the study, a large portion of liquid in the tank is superheated. Therefore, the above analyses are meaningful only if there is no boiling in the liquid, i.e., the maximum temperature, $\mathrm{T}_{\max }$, is less than the incipient boiling temperature. A practical application 
of this study is to predict the required subcooling for a given heat flux so
that bulk boiling can be avoided.

\section{CONCLUSIONS}

This paper presents a numerical study of buoyancy-induced convection in a partially-filled cylindrical tank subjected to wall heat-flux. The liquidvapor interface is assumed flat, shear free, and at a constant saturation temperature. The tank bottom is kept at a constant temperature lower than the interface temperature. Several parameters governing the fluid and thermal behavior are identified and their effects on the velocity and temperature fields are investigated. The results of the investigation based on the parameter ranges covered in the present study are summarized as follows:

1. For a uniform heat flux, the flow pattern usually contains a main counter-clockwise vortex carrying superheated liquid. Also, a weak clockwise vortex is formed in the tank bottom region near the centerline. In the tank bot tom region, heat conduction is generally dominant because the fluid velocity is very low.

2. The energy ratio, $\overline{\mathrm{Nu}}_{\mathrm{b}} /(4 \mathrm{Ar} \mathrm{Nh})$, is a fairly weak function of $\mathrm{Ra}^{*}$ in the range considered in the study. The energy ratio decreases as $\mathrm{Pr}$ increases.

3. Maximum liquid temperature occurs at the upper portion of the tank sidewall. For a given $\mathrm{Nh}$ and $\mathrm{Ar}$, the maximum liquid temperature decreases with an increase in $\mathrm{Ra}^{*}$ and $\mathrm{Pr}$.

4. An increase in $\mathrm{Nh}$ and $\mathrm{Ar}$ results in an increase in the value of maximum liquid temperature and a decrease in the energy ratio, $\overline{\mathrm{Nu}}_{\mathrm{b}} /(4 \mathrm{Ar} \mathrm{Nh})$. Generally, the liquid near the interface region is superheated over the entire tank cross-section. However, if $\mathrm{Ar}$ is too small, only a small region confined in the upper right corner can be superheated and the energy ratio may be greater than one.

5. If $R_{q}$ is large enough, the maximum liquid temperature is located at the upper portion of the tank sidewall. As $R_{q}$ decreases, the value of the maximum temperature decreases while a second temperature peak occurs at the lower portion of the tank sidewall. Further decrease in $R_{q}$, the upper temperature peak disappears and the lower temperature peak increases and becomes the maximum temperature of the liquid. As a result, the value of $\overline{\mathrm{Nu}}_{\mathrm{b}} /(4 \mathrm{Ar} \mathrm{Nh})$ increases with decreasing $R_{q}$ due to the mixing between the convection boundary layer and bulk liquid.

\section{REFERENCES}

1. Bullard, B.R., "Liquid Propellant Thermal Conditioning System Test Program," LMSC-D159262, Lockheed Missiles and Space Co., Sunnyvale, CA, NASA CR-72971,

2. Aydelot t, J.C., Carney, M.J., and Hochstein, J.I., "NASA Lew is Research Center Low-Gravity Fluid Management Technology Program," NASA TM-87145, 1985. 
3. Syed, S.A., Chiappetta, L.M., and Gosman, A.D., "Error Reduct ion Program," PWA-5928-25, Pratt and Whitney Aircraft Co., East Hartford, CT, NASA CR-174776, 1985 .

4. Issa, R.I., "Solution of the Implicitly Discretised Fluid Flow Equations by Operator-Splitting," Journal of Computational Physics, Vol. 62, No. 1 , Jan. 1986, pp. 40-65.

5. Ghia, K.N., Hankey, W.L., Jr., and Hodge, J.K., "Study of Incompressible Navier-Stokes Equations in Primitive Variables Using Implicit Numerical Technique," 3rd Computational Fluid Dynamics Conference, AIAA, 1977. pp. 156-167. (AIAA Paper 77-648).

6. Roberts, G.0., "Computational Meshes for Boundary Layer Problems," Proceedings of the Second International Conference on Numerical Methods in Fluid Dynamics, Lecture Notes in Physics, Vol, 8, J. Ehlers, K. Hepp, and H.A. Weidenmuller, eds., Springer-Verlag, New York, 1971, pp. 171-177.

7. Evans, L.B., Matulevicius, E.S., and Pan, P.C., "Reduction of Thermal Stratification by Nonuniform Wail Heating," AIChE Journal, Vol. 17, No. 3 , May 1971 , pp. 752-753.

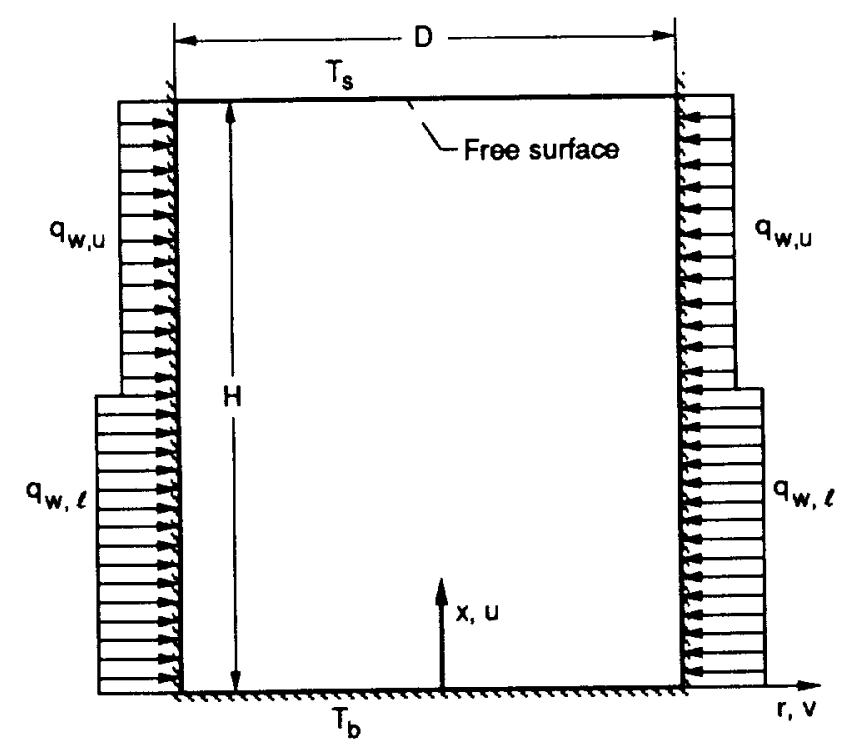

Figure 1.-Physical system and coordinates. 

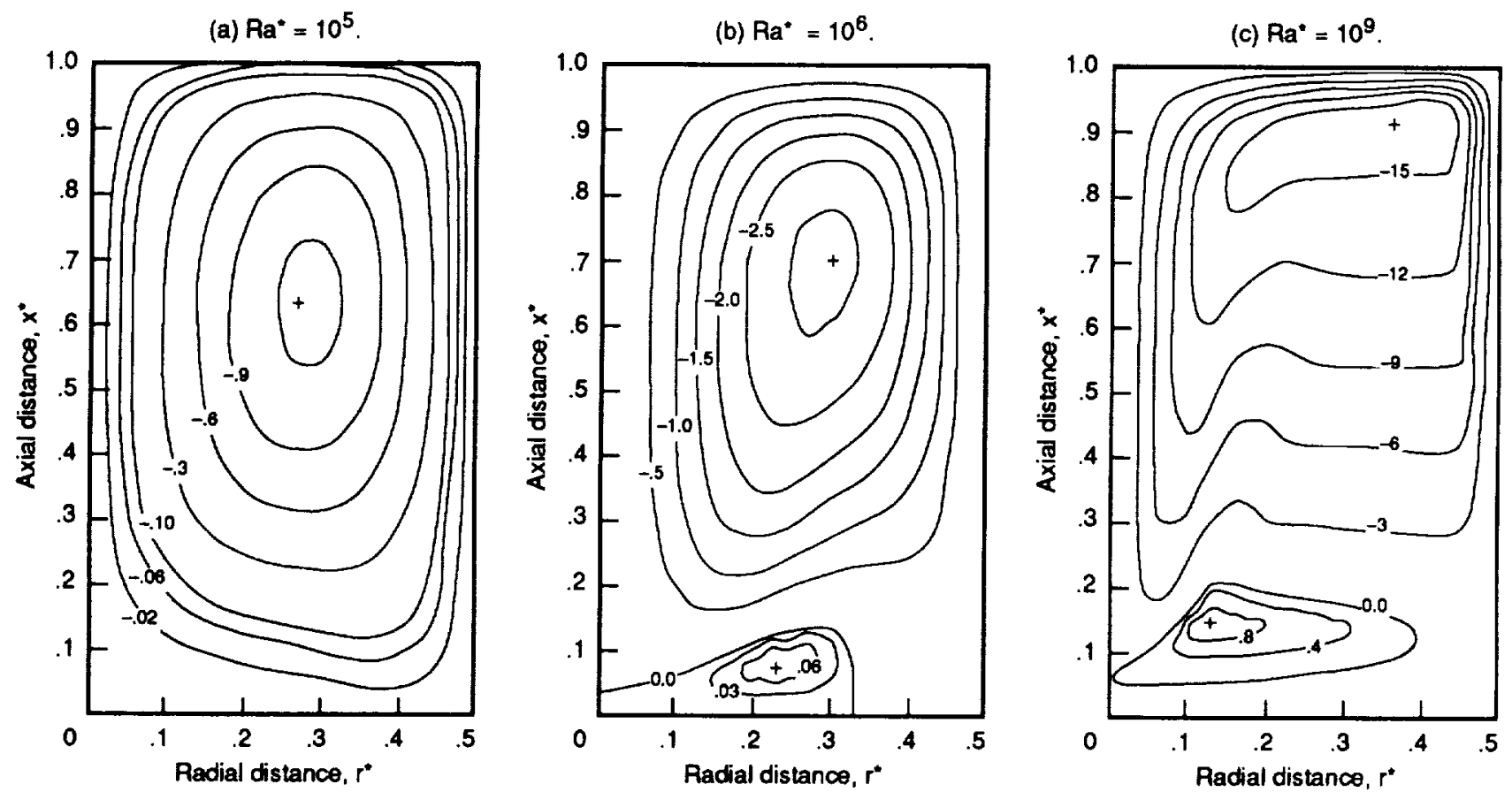

Figure 2. Streamline plot for various $\operatorname{Ra} a^{*}$. $(A r=1.0, \operatorname{Pr}=1.25$, $\mathrm{Nh}=4$ and $\mathrm{Rq}=1.0)$

(a) $R a^{*}=10^{5}$.

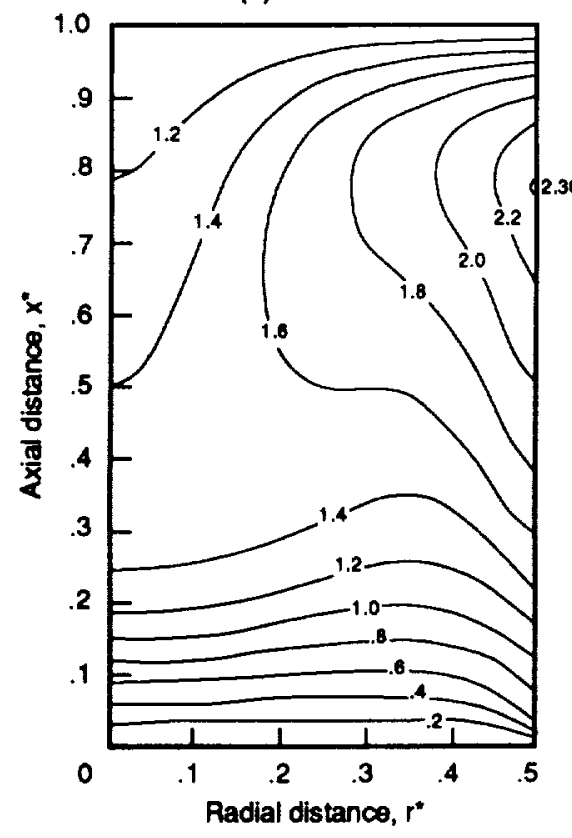

(b) $R a^{*}=10^{6}$.

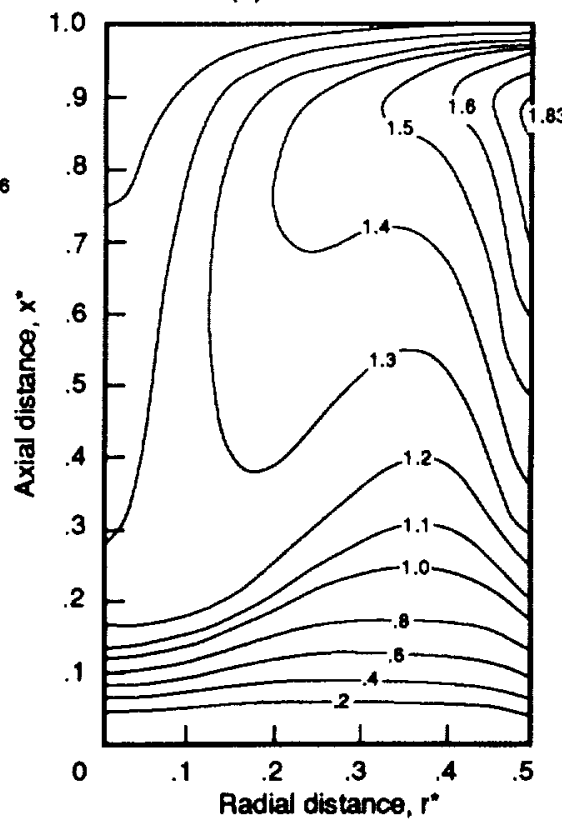

(c) $\mathrm{Ra}^{*}=10^{9}$.

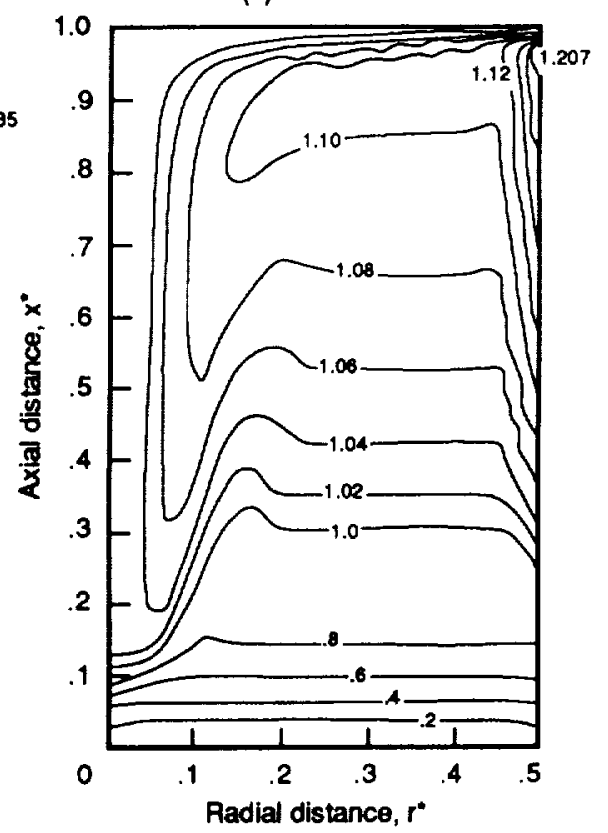

Figure 3.-Isotherm plot for various $\mathrm{Ra}^{*} .(\mathrm{Ar}=1.0, \operatorname{Pr}=1.25, \mathrm{Nh}=4.0$ and $\mathrm{Rq}=1.0$ ) 


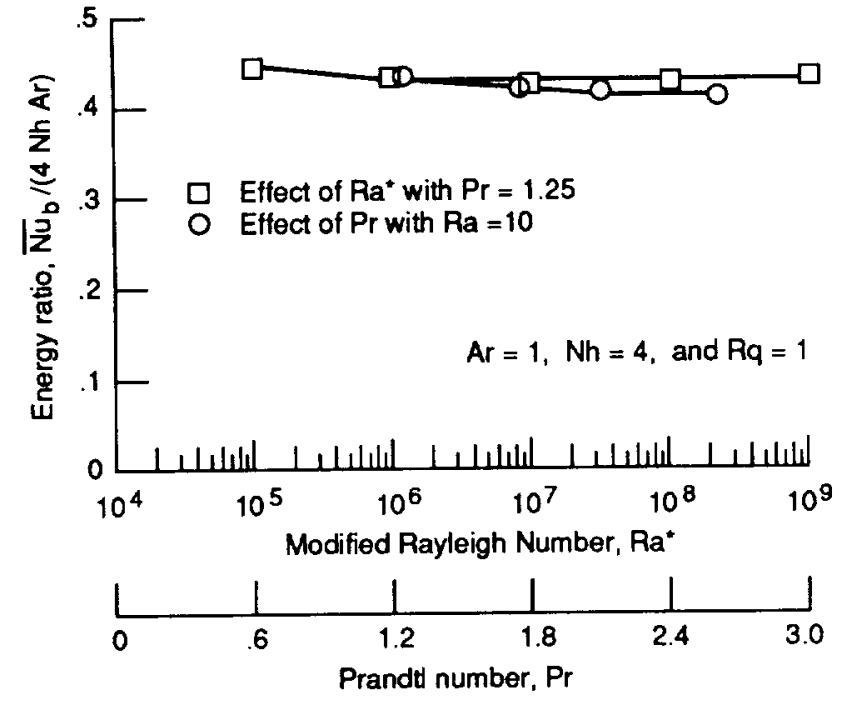

Figure 4.-Effect of Rayleigh Number and Prandt Number on the fraction of the incoming energy transferred through the cold surface.

(a) Streamline

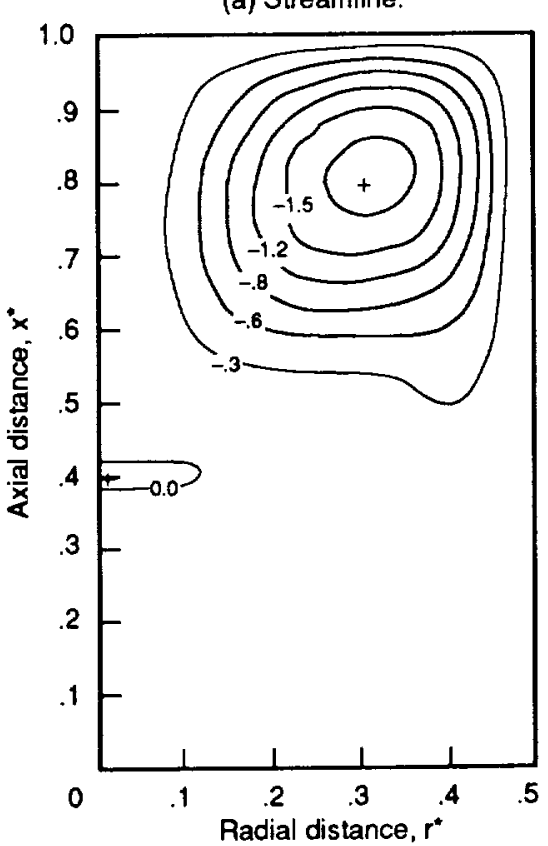

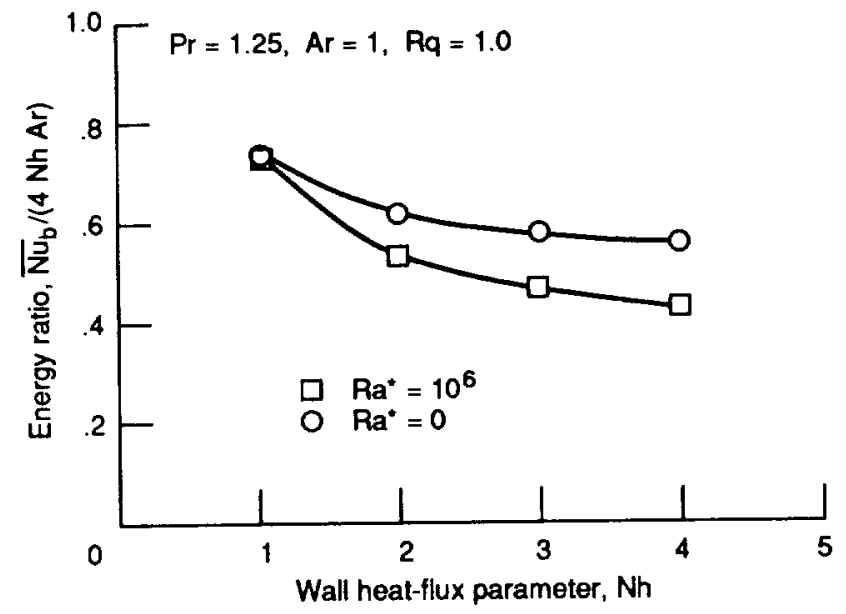

Figure 5.-Effect of wall heal-flux parameter on the fraction of the incoming energy transferred through the cold surface.

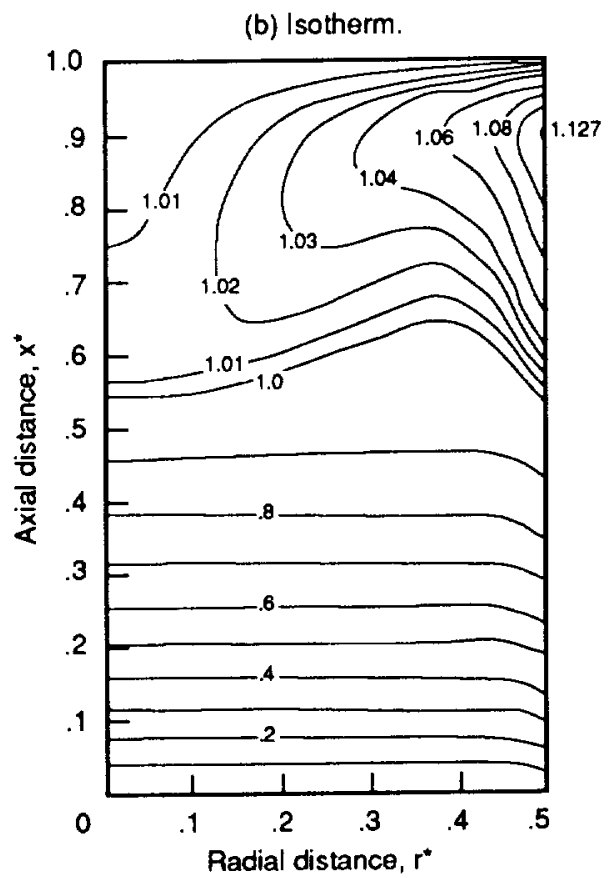

Figure 6. Streamline and isotherm plot for $\mathrm{Aq}=1, \mathrm{Ar}=1, \mathrm{Ra}^{*}=10^{6}, \operatorname{Pr}=1.25$, and $\mathrm{Nh}=1$. 
(a) $\mathrm{Ar}=0.25$.

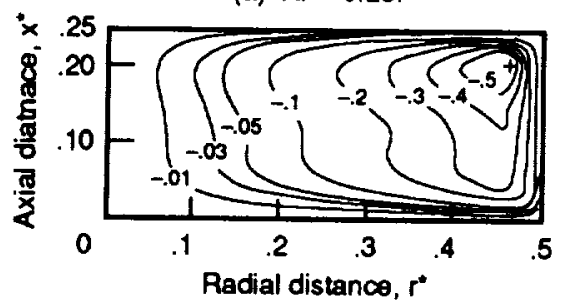

(b) $\mathrm{Ar}=0.50$.

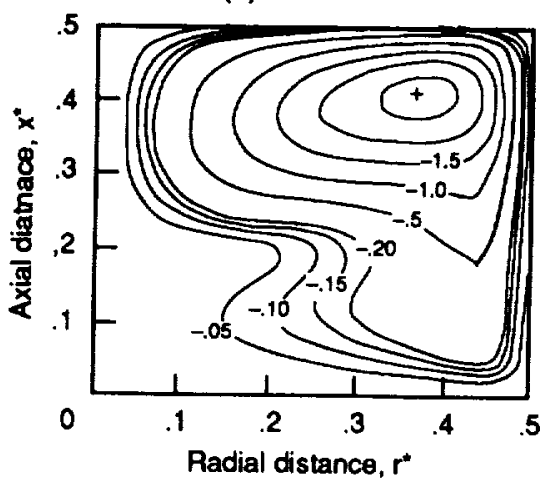

(c) $\mathrm{Ar}=0.75$

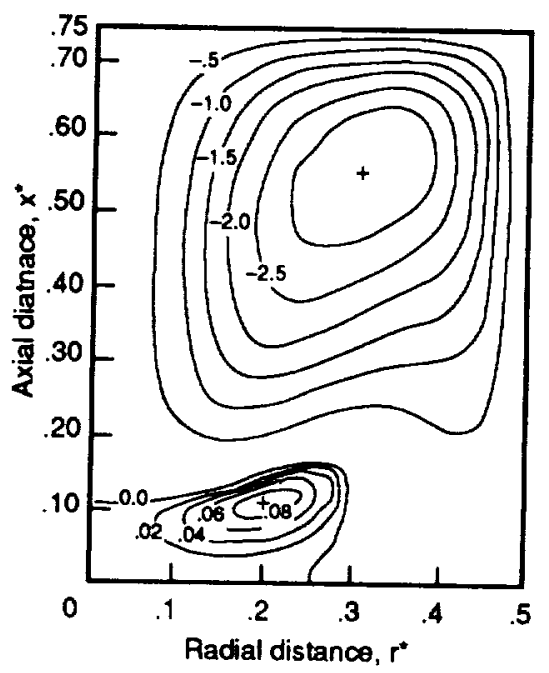

(d) $\mathrm{Ar}=1.25$.

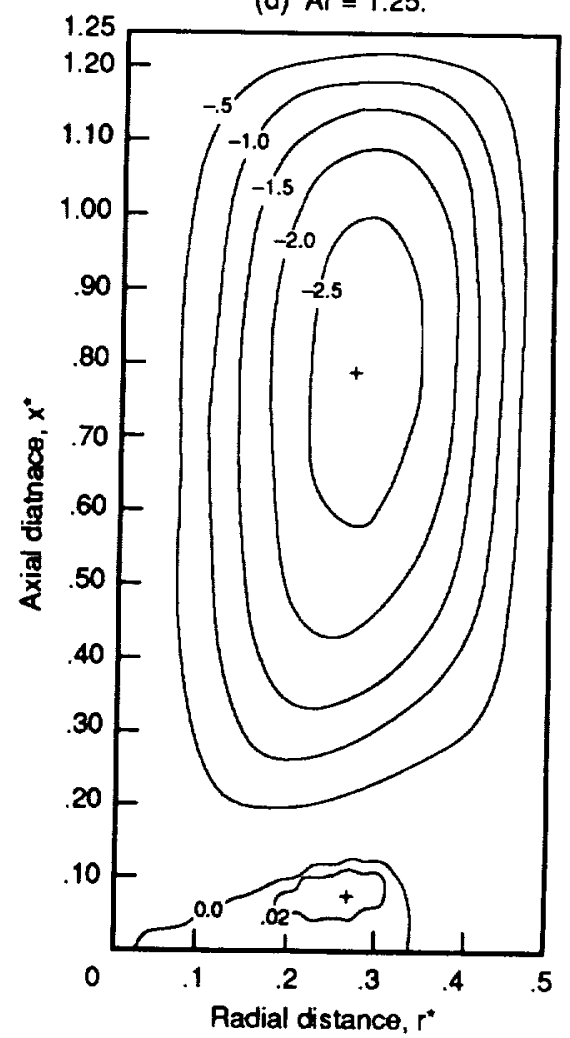

Figure 7.-Streamline plot for various $\operatorname{Ar}$. $\left(\mathrm{Ra}^{*}=10^{6}, \operatorname{Pr}=1.25, \mathrm{Nh}=4\right.$, and $\left.\mathrm{Rq}=1.0\right)$

(a) $\mathrm{Ar}=0.25$.

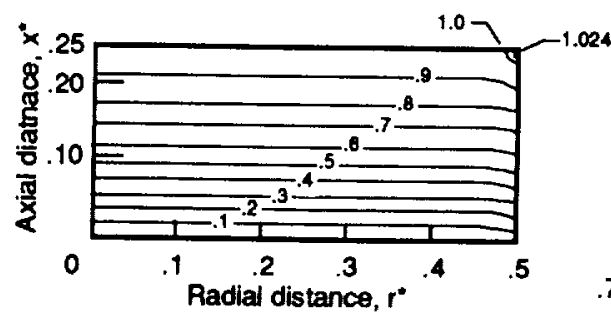

(c) $\mathrm{Ar}=0.75$.

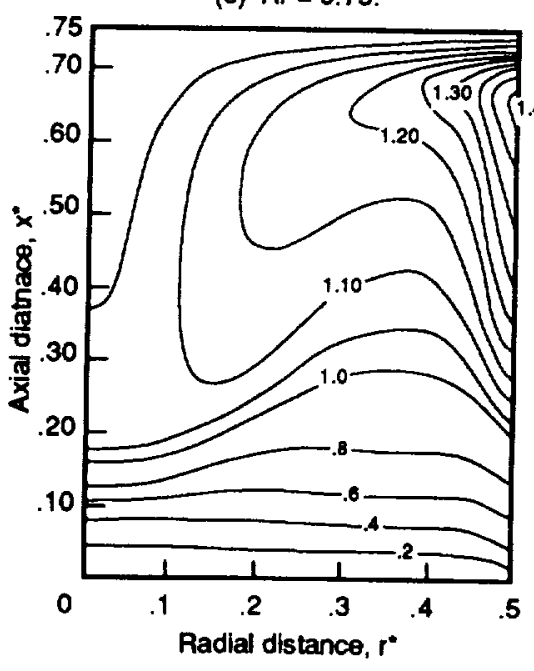

(d) $\operatorname{Ar}=1.25$.

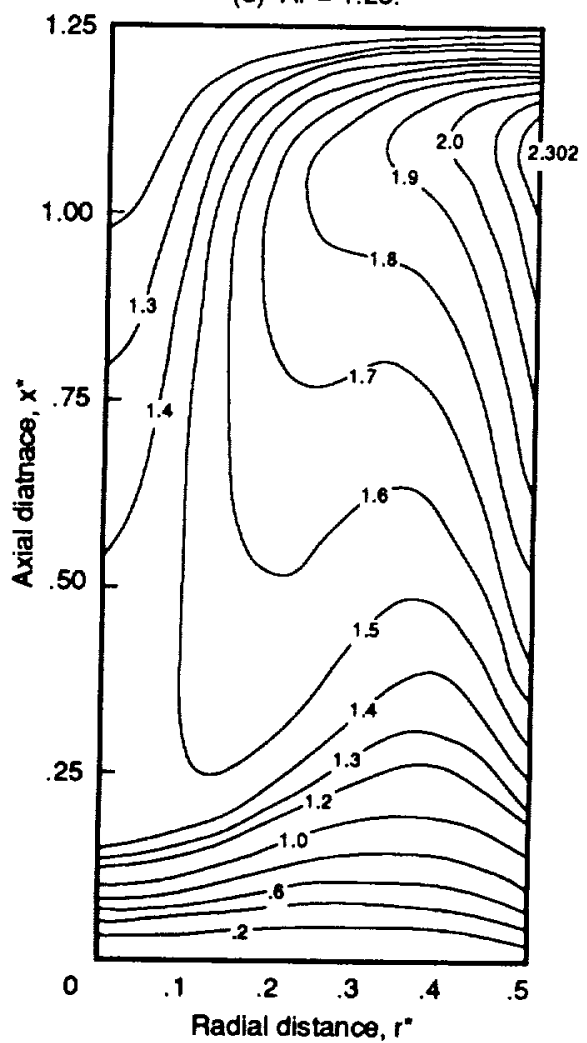

Figure 8. - isotherm plot for various $\mathrm{Ar}$. ( $\mathrm{Ra}^{*}=10^{6}, \mathrm{Pr}=1.25, \mathrm{Nh}=4.0$, and $\mathrm{Rq}=1.0$ ) 


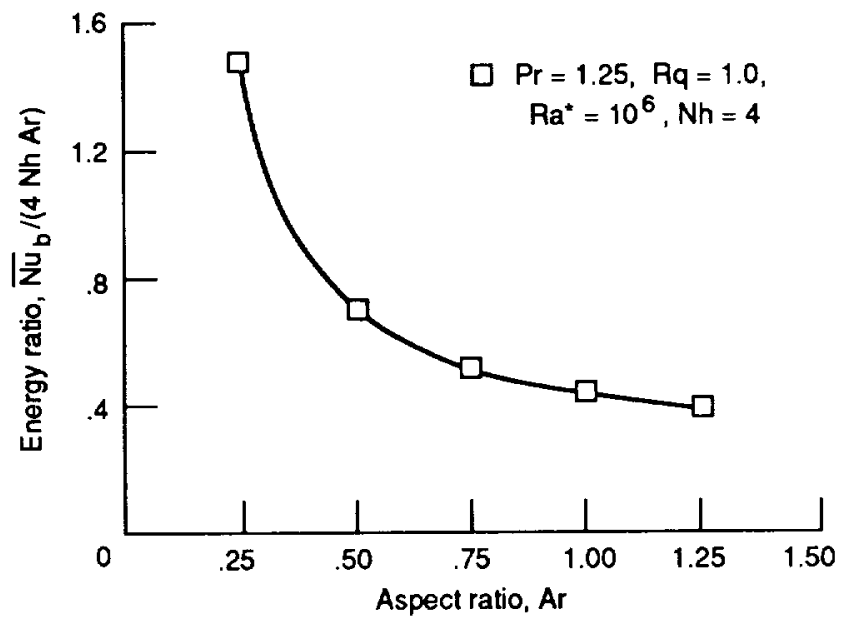

Figure 9.-Effect of aspect ratio on the fraction of the incoming energy transferred through the cold surface.

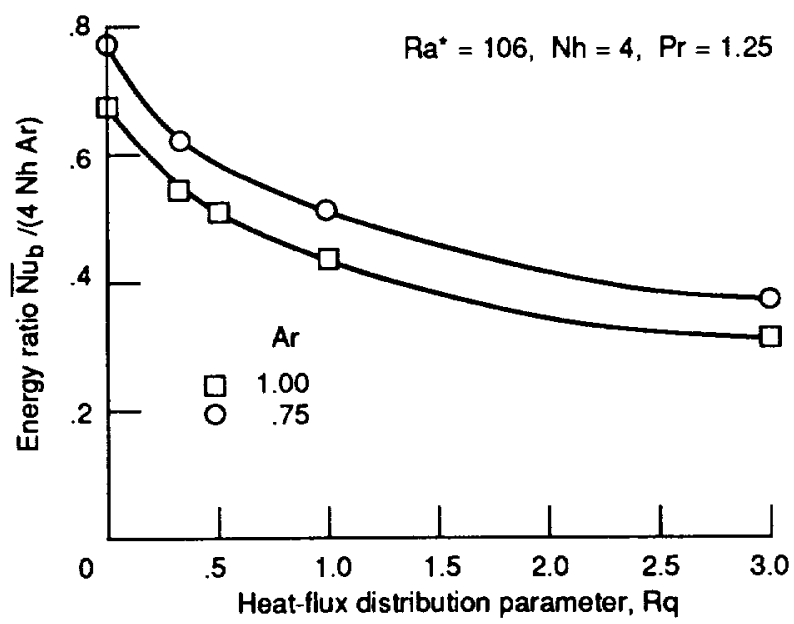

Figure 10-Effect of wall heat-flux distribution parameter on the fract incoming energy transferred through cold surface. 
(a) $\mathrm{Rq}=1 / 3$.

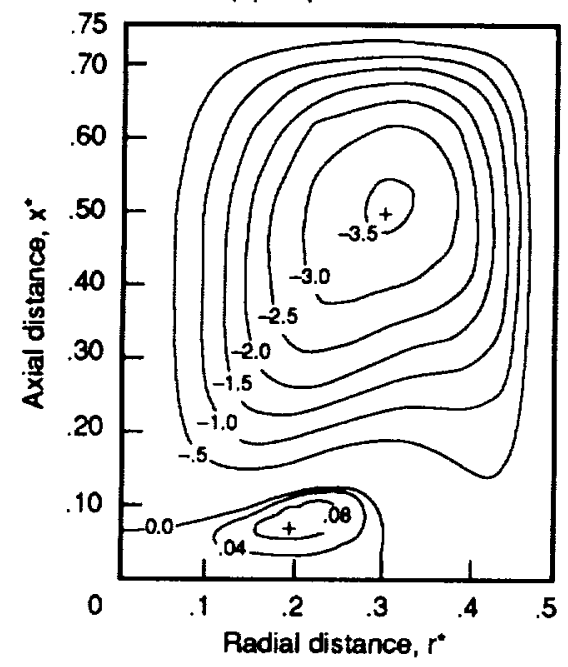

(b) $R q=3$

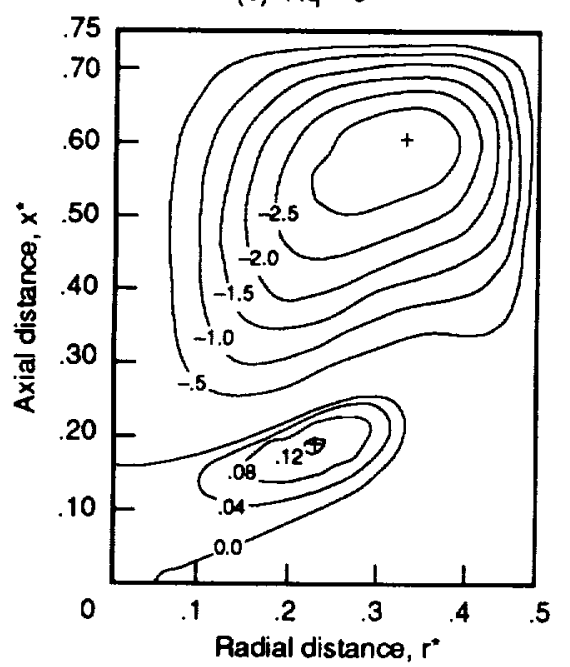

Figure 11. Streamline plot for $\mathrm{Rq}=1 / 3$, and 3. $\left(\mathrm{Ar}=0.75, \mathrm{Ra}^{*}=10^{6}, \mathrm{Pr}=1.25\right.$, and $\mathrm{Nh}=4$ )

(a) $R q=1 / 3$.

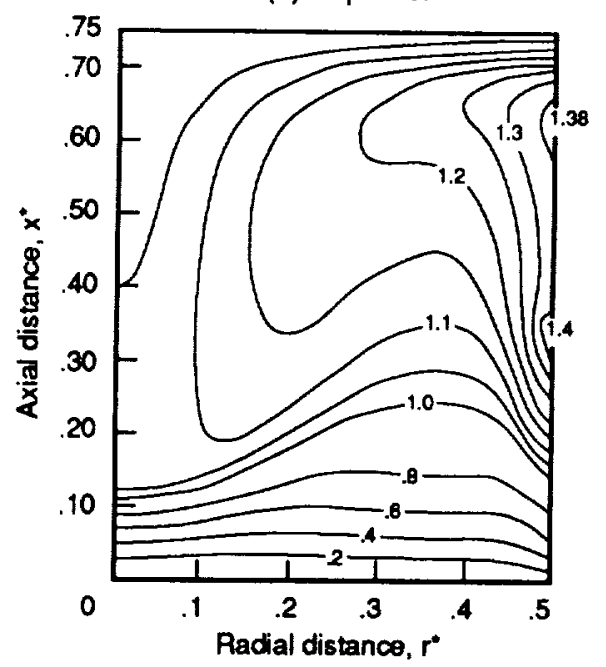

(b) $\mathrm{Rq}=3$

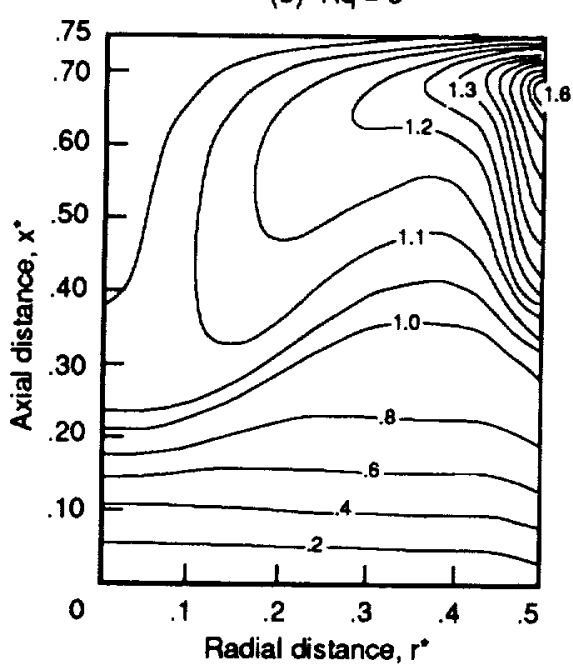

Figure 12.-Isotherm plot for $R q=1 / 3$, and 3. $\left(A r=0.75, R a^{*}=10^{6}, \operatorname{Pr}=1.25\right.$, and $\left.N h=4\right)$ 


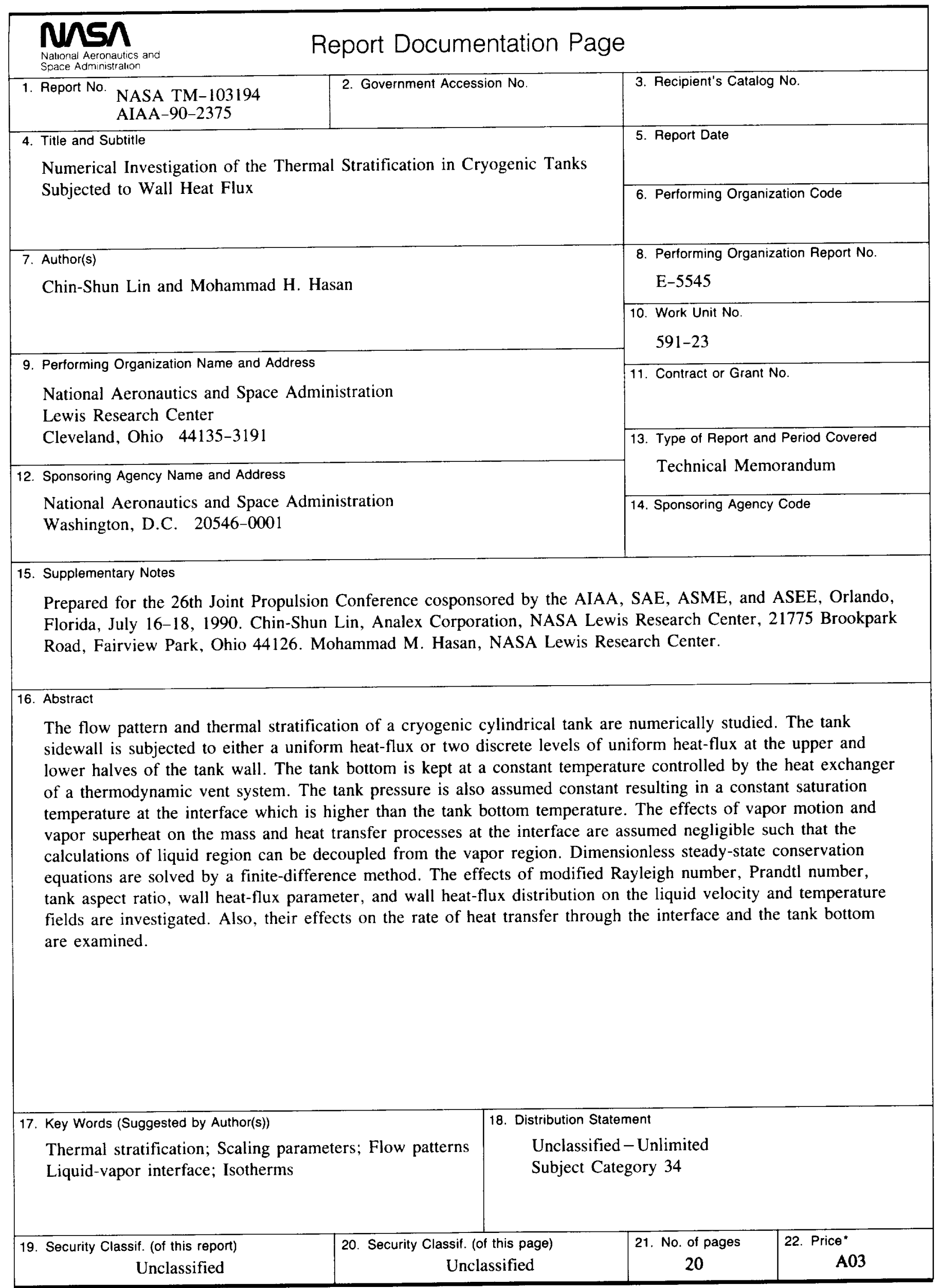



National Aeronautics and

Space Administration

Lewis Research Center

Cleveland, Ohio 44135

Otticiel Business

Penalty for Privale Use $\mathbf{5 3 0 0}$

Poslagond Fees Par

National Aoronaldus and

Space Admonistration

NASA 45 